\title{
Characterization of different osteoclast phenotypes in the progression of bone invasion by oral squamous cell carcinoma
}

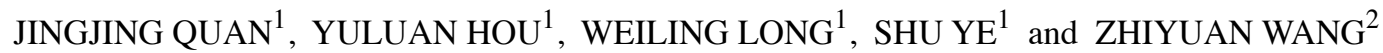 \\ ${ }^{1}$ Guanghua School of Stomatology, Hospital of Stomatology, Sun Yat-sen University \\ and Guangdong Provincial Key Laboratory of Stomatology, Guangzhou, Guangdong 510080; \\ ${ }^{2}$ Affiliated High School-South China Normal University, Guangzhou, Guangdong 510630, P.R. China
}

Received June 18, 2017; Accepted December 5, 2017

DOI: $10.3892 /$ or.2017.6166

\begin{abstract}
The present study aimed to characterize different phenotypes of osteoclasts in the progression of bone invasion by oral squamous cell carcinoma (OSCC). A local bone invasion model of OSCC was established by injecting SCC25 human OSCC cells into the center of calvariae in nude mice, and all mice were found to have a typical bone resorption area. Staining for tartrate-resistant acid phosphatase (TRAP) revealed various types of giant osteoclasts in the tumour-bone interface. Bone marrow cells (BMCs) were isolated from the nude mice for primary osteoclast culture, but only a few giant osteoclasts were generated. Additionally, special blood centrifuge tubes were utilized to obtain large numbers of peripheral blood mononuclear cells (PBMCs). Using magnetic activated cell sorting (MACS) and the cytokines colony-stimulating factor (CSF) and receptor activator of nuclear factor- $\kappa \mathrm{B}$ ligand (RANKL), we differentiated human osteoclasts from $\mathrm{CD} 14^{+}$ monocytes of PBMCs. Bone resorption was further confirmed by a bone resorption assay. Finally, Transwell inserts were used for indirect cell co-culture of SCC25 cells and $\mathrm{CD} 14^{+}$monocytes. Expression of specific osteoclast markers was detected by real-time PCR and western blotting. After co-culture for 3 and 6 days, conditioned medium (CM) of SCC25 cells stimulated the expression of osteoclast markers, and additional osteoclasts were detected through staining of TRAP and F-actin. In the present study distinct osteoclast phenotypes were observed in the established bone invasion animal model, and were confirmed using various primary osteoclast cultures. CM of OSCC cells may promote the expression of osteoclast markers and induce the differentiation of monocytes to mature osteoclasts, which can resorb adjacent bone tissue.
\end{abstract}

Correspondence to: Dr Jingjing Quan, Guanghua School of Stomatology, Hospital of Stomatology, Sun Yat-sen University and Guangdong Provincial Key Laboratory of Stomatology, Guangzhou, Guangdong 510080, P.R. China

E-mail: quanjj3@mail.sysu.edu.cn

Key words: osteoclasts, bone invasion, oral squamous cell carcinoma, magnetic activated cell sorting

\section{Introduction}

Osteoclasts, derived from the mononuclear phagocyte system, are the main cells capable of resorbing bone (1). Physiologically, bone homeostasis is maintained by the balance between osteoblastic bone formation and osteoclastic bone resorption (2). However, excess mass bone resorption and an imbalance in weight are observed in various osteolytic diseases, including arthritis, periodontitis, loosened tooth implants and malignancy-induced bone metastasis $(3,4)$. Regardless of disease type, osteoclasts play important roles in the body. Once osteoclasts are recruited to bone sites, the bone architecture or function becomes abnormal (5).

In contrast to distant bone metastasis caused by breast or prostate cancer, local bone invasion is typically detected in oral cancer, particularly OSCC. Bone invasion by OSCC exhibits specific characteristics, which depend on crosstalk between osteoblasts, osteoclasts and tumour cells (6). Our research group focused on these complex interactions. By using an indirect cell co-culture model, we found that $\mathrm{CM}$ from both an osteoblast cell line and primary cultured osteoblasts stimulated the invasive properties of OSCC cells, while CM of OSCC cells induced the generation of osteoclastic factors in osteoblasts (7). To determine which component in the CM caused gene expression changes during cell co-culture, transforming growth factor- $\beta$ (TGF- $\beta$ ) was utilized to treat OSCC cells, and partial epithelial-mesenchymal transition (EMT) was confirmed in OSCC cells. CM of OSCC cells pre-treated with TGF- $\beta$ promoted the survival of mature osteoclasts (8). In addition, we demonstrated that specific inhibition of monocyte chemoattractant protein-1 (MCP-1) in OSCC cells by transfection with by 7 amino acids truncated (7ND), reduced osteoclast differentiation in vitro, and the bone invasion area within the calvariae of nude mice (9). These studies revealed molecules with the potential to inhibit bone invasion for future biotherapy.

In the present study, we used a calvaria bone invasion mouse model of OSCC, to examine how osteoclast precursors are recruited by tumour cells. First, we isolated BMCs from nude mice and started primary osteoclast culture, which generated only a few osteoclasts. Therefore, we used PBMCs and specific blood centrifuge tubes to obtain large numbers of monocytes in vitro. By MACS and the cytokines CSF and 
RANKL, human osteoclasts were differentiated from CD14 ${ }^{+}$ monocytes of PBMCs. Bone resorption function was further explored. Finally, Transwell inserts were utilized for indirect cell co-culture between human OSCC cells and $\mathrm{CD}_{14}{ }^{+}$monocytes. Expression of specific osteoclast markers were detected by real-time PCR and western blotting. The results suggested that CM of tumour cells can induce the expression of osteoclast markers, and differentiation of monocytes to mature osteoclasts to resorb adjacent bone.

\section{Materials and methods}

Reagent. Dulbecco's modified Eagle's medium (DMEM), $\alpha$-MEM, fetal bovine serum (FBS), trypsin-EDTA, antibiotics and phosphate-buffered saline (PBS) were purchased from Thermo Fisher Scientific (Waltham, MA, USA). The primary antibodies were mouse anti-human monoclonal matrix metalloproteinase-9 (MMP-9) and anti-cathepsin K (CTSK; Santa Cruz Biotechnology, Santa Cruz, CA, USA), rabbit anti-human polyclonal nuclear factor of activated T cell 1 (NFATc1; Cell Signaling Technology, Danvers, MA, USA), and mouse anti-human monoclonal $\alpha$-tubulin (Abcam, Cambridge, UK). The horseradish peroxidase (HRP)-conjugated secondary antibody was supplied by Bio-Rad Laboratories (Hercules, CA, USA). Recombinant murine and human cytokines CSF and RANKL were obtained from PeproTech (Rocky Hill, NJ, USA). The TRAP staining kit was from Sigma-Aldrich (St. Louis, MO, USA). The OSCC cell line SCC25 was obtained from the American Type Tissue Collection (ATCC; Manassas, VA, USA), and was maintained in DMEM with $10 \%$ FBS and antibiotics (100 U/ml of penicillin $\mathrm{G}$ and $100 \mathrm{mg} / \mathrm{ml}$ of streptomycin) at $37^{\circ} \mathrm{C}$ in an incubator $\left(5 \% \mathrm{CO}_{2} / 20 \% \mathrm{O}_{2}\right)$.

Establishment of animal model of bone invasion using SCC 25 cells. BALB/c nude mice were purchased from the animal resources center, housed in an animal facility and cared for by animal house staff. All protocols were reviewed and approved by the university ethics committee (2016-334QX). At 6-7 weeks of age, the mice were utilized to develop an animal model of bone invasion by OSCC (9). Under sterile condition, SCC25 $\left(5 \times 10^{6}\right.$ cells $\left./ 100 \mu 1\right)$ were injected subcutaneously overlaying the calvaria. Body weight and tumour volume were recorded each week. All animals were sacrificed at week 4, and BMCs were obtained immediately for subsequent cell culture experiments.

Micro-computed tomography $(\mu C T)$ imaging. All calvariae were surgically removed from SCC25 tumour-bearing nude mice, fixed in $70 \%$ ethanol and scanned using a $\mu \mathrm{CT}$ instrument (Scanco Medica AG, Brüttisellen, Switzerland). $\mu$ CT-analyzer software was used to analyze the structure of the calvaria using a global segmentation method. Two-dimensional images were used for three-dimensional reconstruction. The resorpted area of each calvaria was determined for analysis and quantification as previously reported (9).

Histological and immunohistochemical analysis. Tumour specimens from nude mice were embedded in paraffin using a tissue processor. Serial $5-\mu \mathrm{m}$ paraffin sections were cut using a rotary microtome (Leica Microsystems, Wetzlar, Germany) and stained with hematoxylin and eosin (H\&E). For tumour-bearing calvaria, all calvariae were decalcified in $10 \%$ EDTA ( $\mathrm{pH}$ 7.4) for 2 weeks and then processed for paraffin embedding. Serial $5-\mu \mathrm{m}$ sections were stained with both $\mathrm{H} \& \mathrm{E}$ and TRAP. TRAP staining was performed using a standard protocol. Giant TRAP-positive cells along the tumour-bone interface were considered osteoclasts.

Primary osteoclast culture from bone marrow mononuclear cells of nude mice. Nude model mice were sacrificed and dissected to obtain humerus and tibia (10). All cell culture experiments were conducted in $\alpha$-MEM supplemented with $10 \% \mathrm{FBS}$ and $1 \%$ penicillin-streptomycin, in a $5 \% \mathrm{CO}_{2}$ atmosphere at $37^{\circ} \mathrm{C}$. BMCs were flushed from the bone with medium and then filtered through a $600-\mu \mathrm{m}$ cell strainer. Cells were seeded in $150 \times 25 \mathrm{~mm}$ cell culture dishes at a density of $1.5 \times 10^{4}$ cells $/ \mathrm{cm}^{2}$ with $30 \mathrm{ng} / \mathrm{ml}$ murine CSF. After 2 days, adherent cells were separated from non-adherent cells and plated in 24-well plates at a density of $1 \times 10^{5}$ cells/well. Osteoclast-like cells were generated by adding murine CSF (30 ng/ml) and RANKL (35 ng/ml). After continuous culture for 10 days, cells were fixed with $10 \%$ formaldehyde solution and TRAP-stained according to the manufacturer's instructions. Comparing with single cells, these round and giant cells, strongly TRAP stained and three or more cell fusion, were considered multinucleated osteoclasts. Four fields were randomly selected and non-overlapping images were taken for each of triplicate culture wells. In each image the total numbers of TRAP-positive multinucleated osteoclasts were counted by two independent assessors.

MACS of $\mathrm{CD}_{14}^{+}$monocytes. Human PBMCs were isolated from the blood of healthy volunteers using BD Vacutainer cell preparation tubes (BD Biosciences, Franklin Lakes, NJ, USA) containing sodium citrate (9). Informed consent was obtained from each patient. After centrifugation at $1,500 \mathrm{x} \mathrm{g}$ for $30 \mathrm{~min}$, the cell layer on top of the Ficoll-Paque was collected, resuspended in $10 \mathrm{ml}$ of $\alpha-\mathrm{MEM}$ and centrifuged $(1,250 \mathrm{rpm}$, $10 \mathrm{~min}) . \mathrm{CD}^{+} 4^{+}$monocytes were purified by incubation with MACS CD14+ MicroBeads (Miltenyi Biotec GmbH, Bergisch Gladbach, Germany) for $15 \mathrm{~min}$ at $4^{\circ} \mathrm{C}$. Cells were then washed with $\mathrm{CD} 14^{+}$isolation buffer $[0.5 \%$ fetal calf serum (FCS), 2 mM EDTA, pH 8.0] and passed through a MACS cell separator. $\mathrm{CD}_{1}{ }^{+}$monocytes were collected and utilized for subsequent experiments.

Osteoclast differentiation assay. $\mathrm{CD}_{14}^{+}$monocytes were plated in 24 -well plates $\left(1 \times 10^{5}\right.$ cells/well) containing $600 \mu 1$ medium ( $\alpha$-MEM, pH 7.4, containing 10\% FBS and $1 \%$ penicillin/streptomycin) in each well. To induce osteoclast differentiation, groups were arranged as follows: Group 1, $\mathrm{CD}_{14}{ }^{+}$monocytes only; Group $2, \mathrm{CD}_{14}{ }^{+}$monocytes with CSF $(25 \mathrm{ng} / \mathrm{ml})$ and RANKL (40 ng/ml). Total medium was changed every 3 days and osteoclasts appeared in 1 week. Osteoclasts were subsequently fixed in $10 \%$ formaldehyde solution. TRAP staining was used to characterize osteoclasts. Comparing with single cells, these round and giant cells, strongly TRAP stained and three or more cell fusion, were considered multinucleated osteoclasts. Four fields were 
randomly selected and non-overlapping images were taken for each of triplicate culture wells. In each image the total numbers of TRAP-positive multinucleated osteoclasts were counted by two independent assessors.

Immunofluorescence. To further confirm staining of the F-actin ring, immunofluorescence (IF) was utilized. Briefly, osteoclasts were fixed in $10 \%$ formaldehyde solution, permeabilized with $0.5 \%$ Triton $\mathrm{X}-100$ and stained in the dark. Rhodamineconjugated phalloidin (Life Technologies, Carlsbad, CA, USA) was used to label F-actin. DAPI staining (Life Technologies) was used to visualize the nuclei. Cells were visualized using a fluorescence microscope. Four fields were randomly selected and counted for the numbers of F-actin rings by two independent assessors.

Bone resorption assay. To confirm bone resorption activity, $\mathrm{CD}^{+}{ }^{+}$monocytes were plated on dentin slices of 96-well plates. After 21 days of culture with cytokines of CSF and RANKL, mature osteoclasts were fixed in acetone, citrate and formaldehyde solution and stained with TRAP. Bone resorption assays were performed on dentine slices as previously described (12). Dentine slices were sputter-coated with gold and observed with a scanning electron microscope. All dentin slices were kindly provided by Dr Nigel A. Morrison from Griffith University (Southport, Gold Coast, Australia).

Indirect cell co-culture between SCC25 cells and CD14+ monocytes. Transwell inserts $(0.4-\mu \mathrm{m}$ pore; Corning, Inc., Corning, NY, USA) were used for indirect cell co-culture. SCC 25 cells $\left(5 \times 10^{3}\right.$ cells/well $)$ were seeded in the upper chamber, and human $\mathrm{CD}_{14}{ }^{+}$monocytes $\left(5 \times 10^{4}\right.$ cells/well $)$ were placed in the bottom of 24-well plates. The chambers were incubated for 3 and 6 days, respectively. Cytokines of CSF and RANKL were added to the bottom of 24-well plates, while the negative control group contained no cytokines. The medium was changed and new cytokine was added every 2 days; both RNA and protein were extracted from monocytes at each of the tested time-points.

Real-time PCR. Total RNA was isolated from monocytes using a PureLink RNA Mini kit (Invitrogen, Carlsbad, CA, USA), and reverse transcribed to cDNA using an iScript cDNA Synthesis kit (Bio-Rad Laboratories) according to the manufacturer's instructions. Quantitative gene analysis was performed for NFATc1, MMP-9 and CTSK using the Express SYBR GreenER qPCR Supermix Universal kit (Invitrogen) and LightCycler 480 Real-time PCR system (Roche). Data were normalized to the internal control GAPDH to obtain $\Delta \mathrm{Cq}$. The fold change in genes of interest relative to untreated samples was determined using the $2^{-\Delta \Delta \mathrm{Cq}}$ method (11). The primer sequences have been previously reported $(12,13)$.

Western blotting. Total protein was extracted from monocytes by using lysis buffer (Thermo Fisher Scientific). Protein concentration was determined with a BCA Protein Assay kit (Pierce, Rockford, IL, USA). Next, $40 \mu \mathrm{g}$ of protein was subjected to SDS-PAGE using $10 \%$ poly-acrylamide gels. Proteins were transferred to polyvinylidene fluoride membranes, and blocked with $5 \%$ non-fat dry milk in Tris-buffered saline
(TBS) for $1 \mathrm{~h}$ at room temperature. The membranes were then incubated with primary antibodies for NFATc1 (1:500), MMP-9 (1:200), CTSK (1:200), and $\alpha$-tubulin (1:3,000) overnight at $4^{\circ} \mathrm{C}$, washed twice, and incubated with horseradish peroxidase-conjugated (HRP) secondary antibodies for $1 \mathrm{~h}$ at room temperature. Protein bands were detected with SuperSignal West Pico Chemiluminescent Substrate (Thermo Fisher Scientific) and visualized using VersaDoc MP Imaging Systems (Bio-Rad Laboratories).

Differentiation of osteoclasts from indirect cell co-culture. After co-culture for 3 and 6 days, $\mathrm{CD} 14^{+}$monocytes were fixed in $10 \%$ formaldehyde solution. TRAP and F-actin staining were performed to characterize the osteoclasts. TRAP-positive cells with three or more nuclei were considered as multinucleated osteoclasts. Rhodamine-conjugated phalloidin was used to stain F-actin, while DAPI was used to stain the nuclei. Four fields were randomly selected and counted for osteoclast and F-actin numbers by two independent assessors.

Statistical analysis. Results were presented as the mean \pm standard error (SE) of at least 3 independent experiments. Data analysis was performed using SPSS Software (version 20.0; SPSS, Inc., Chicago, IL, USA). Student's t-test was used to compare two groups. One-way analysis of variance was applied to compare two or more groups, followed by StudentNewman-Keuls test. A $\mathrm{P}<0.05$ was regarded as statistically significant.

\section{Results}

Typical bone resorption in calvarial bone invasion model of OSCC. By injecting SCC25 cells into the center of calvariae in nude mice, a local bone invasion animal model of OSCC was established (Fig. 1A). All 6 nude mice showed typical bone resorption areas (Fig. 1B). No mice died during the experiment.

Various types of giant osteoclasts in the tumour-bone interface. Histological analysis of tumour samples from the animal model suggested that the formation of squamous cell carcinoma in vivo, which invaded adjacent bone tissue (Fig. 2A). TRAP staining revealed various types of giant osteoclasts in the tumour-bone interface, displaying different phenotypes including round, triangle, or slabstone shapes (Fig. 2B).

Few osteoclasts were generated from BMCs of nude mice. A primary osteoclast culture from BMCs was established in vitro. BMCs were obtained from nude mice as previously reported (10). However, after continuous culture for 10 days, few osteoclasts were generated in each of the 6 nude mice. TRAP staining observed several round and giant osteoclast precursors (Fig. 3A). Normal control and tumour-bearing nude mice showed similar results (Fig. 3B).

Special centrifuge blood tubes for monocyte collection and primary human osteoclast culture by MACS. Special centrifuge blood tubes were utilized to enrich human PBMCs (Fig. 4A), which were further labeled with a CD14 ${ }^{+}$magnetic antibody and flowed through a MACS selector (Fig. 4B). In the 

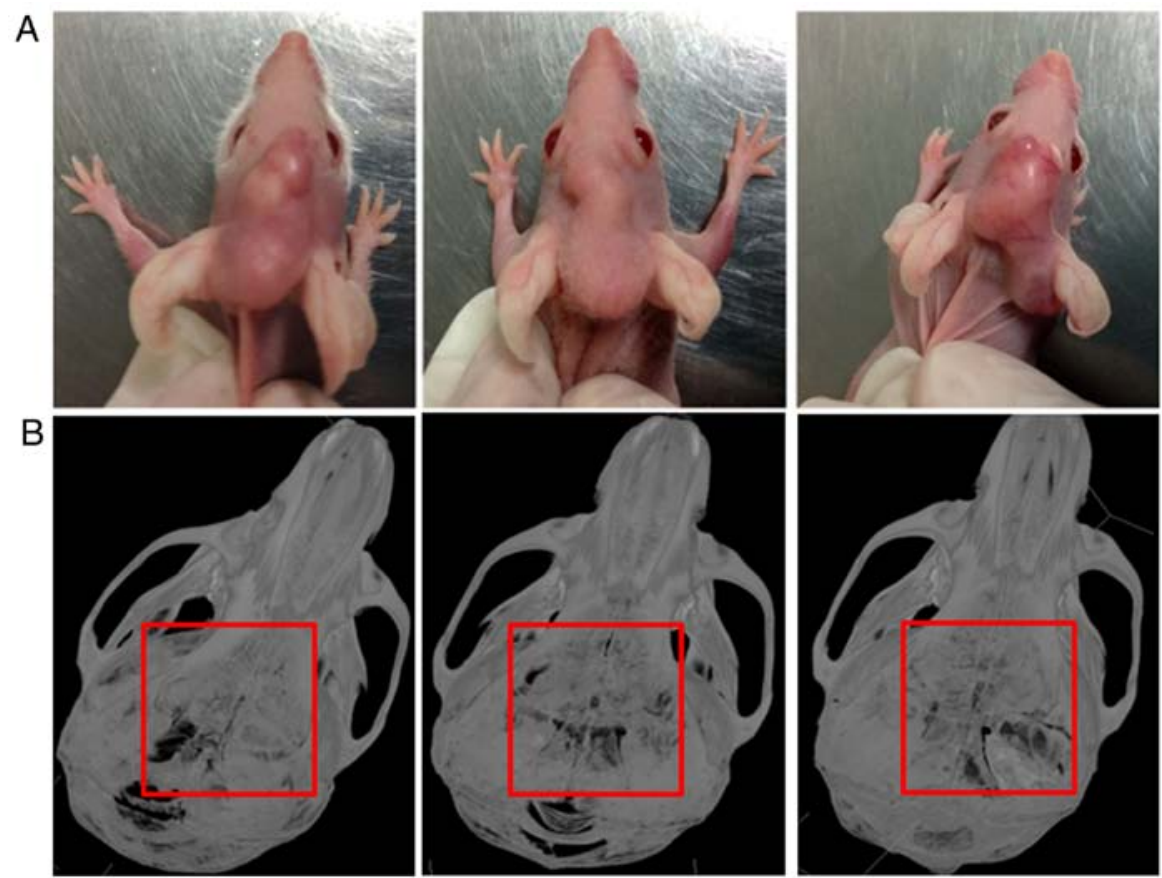

Figure 1. Typical bone resorption was observed in calvarial bone invasion model of OSCC. (A) SCC25 cells were injected through the center of the calvaria, and the tumour formed in 1 month. (B) All nude mice showed typical bone resorption according to uCT analysis. Representative photos of the tumour formed and the bone invasion area.
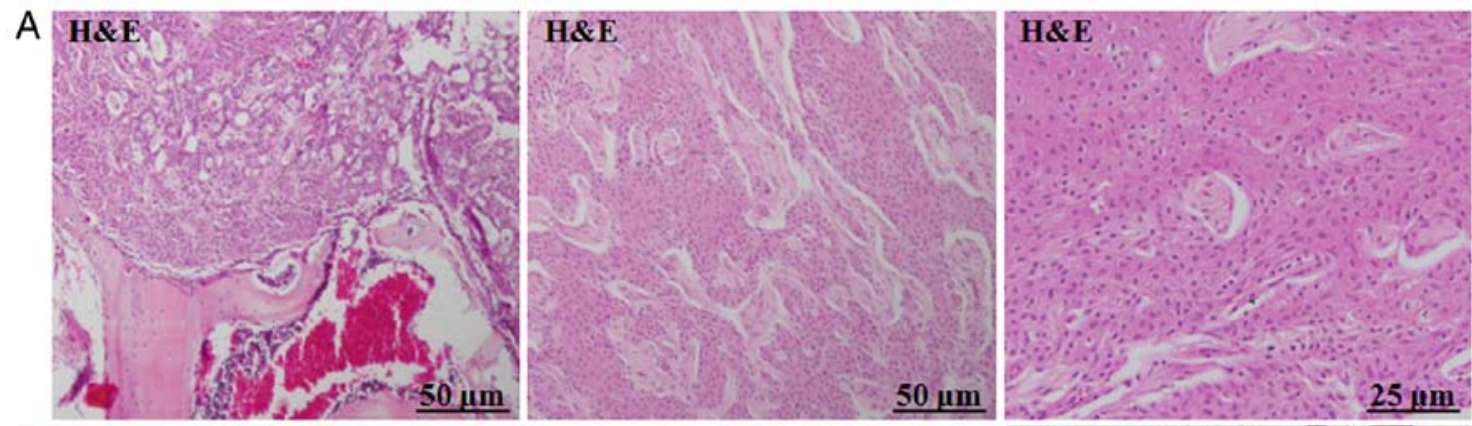

$\mathrm{B}$
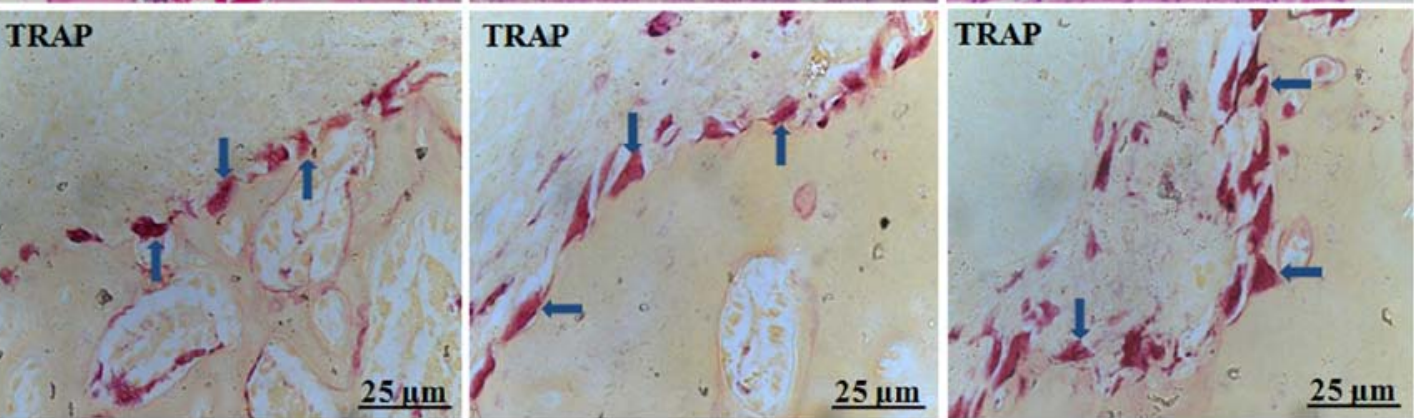

Figure 2. Various types of giant osteoclasts were observed at the tumour-bone interface in the animal model. (A) H\&E staining suggested the formation of squamous cell carcinoma in vivo, which invaded adjacent bone tissue; (B) TRAP staining revealed various types of giant osteoclasts and different phenotypes including round, triangle, or slabstone shapes (arrow, TRAP). Representative photos of TRAP staining.

presence of CSF and RANKL, osteoclasts differentiated after continuous culture for 6 days. TRAP staining indicated typical round and giant osteoclasts and IF confirmed the formation of F-actin ring (Fig. 4C).

Scanning electron microscopy (SEM) confirmed bone resorption pits of dentin slides degraded by osteoclasts. To further confirm whether the obtained osteoclasts could resorb bone,
$\mathrm{CD} 14^{+}$monocytes were implanted on dentin slides and cultured for 21 days. TRAP staining of both cells with or without dentin slides suggested normal TRAP staining (Fig. 5A). SEM further confirmed the typical osteoclast morphology (Fig. 5B). Negative control slides showed $\mathrm{CD} 14^{+}$monocytes only (Fig. 5B). Different magnifications were utilized to observe resorption pits, which were in the forms of either single, small resorption tracks or discrete areas of lacunar excavations 

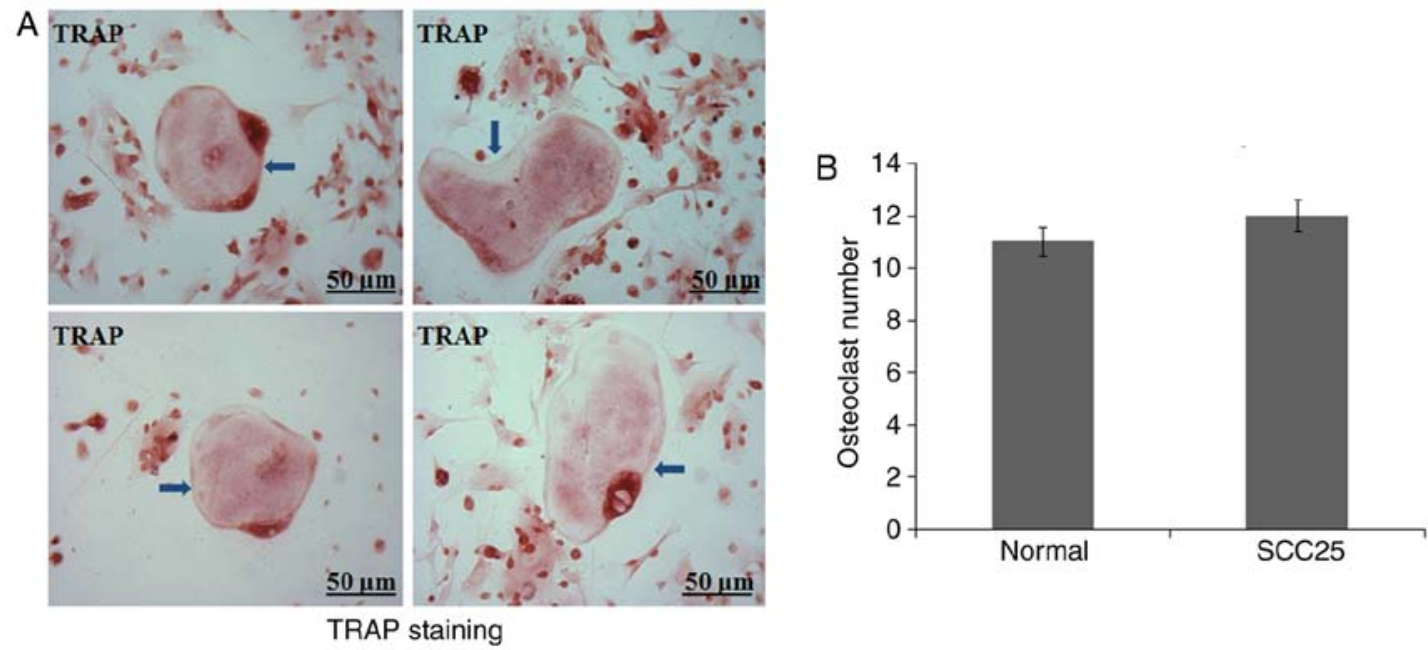

Figure 3. Few osteoclasts were generated from BMCs from nude mice. (A) After continuous culture for 10 days, few osteoclasts were differentiated. TRAP staining showed several round and giant osteoclast precursors (arrow, TRAP). Representative photos of TRAP staining for osteoclasts. (B) Quantification of osteoclast numbers between normal control and tumour-bearing nude mice. No significant differences were found between groups $(P>0.05)$.
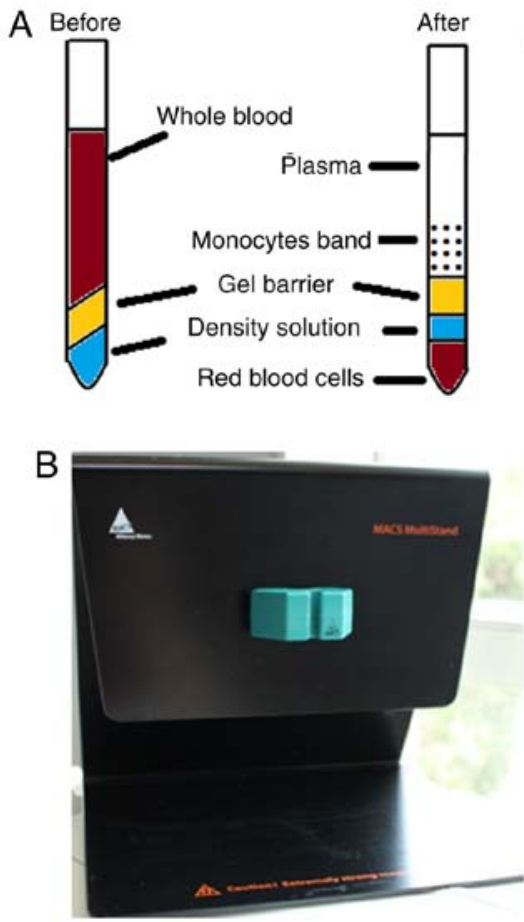
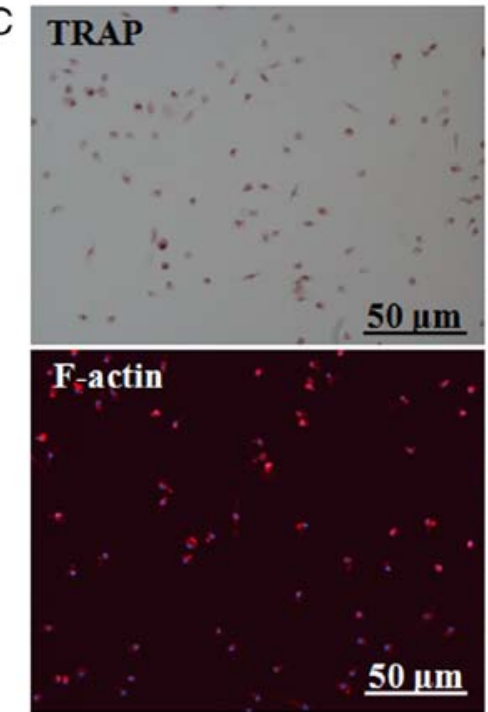

CD14+ monocytes
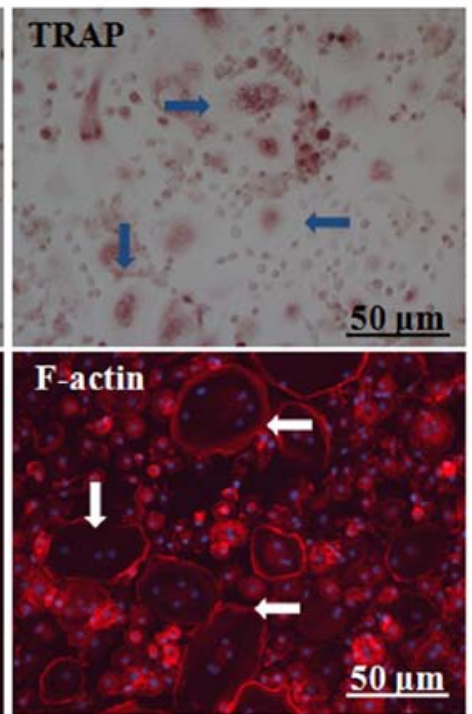

CD14+ monocytes with CSF and RANKL

Figure 4. Special centrifuge blood tubes were utilized for monocyte collection and primary human osteoclast culture by MACS. (A) Schematic diagram of the centrifuge blood tube; (B) MACS selector; (C) TRAP staining indicated that typical round and giant osteoclasts were differentiated (arrow, TRAP), and IF confirmed the formation of F-actin rings (arrow, F-actin). Representative photos of osteoclasts.

(Fig. 6A). No resorption pits were observed in the control group containing only $\mathrm{CD} 14^{+}$monocytes (Fig. 6B).

Expression changes in osteoclast markers detected by realtime PCR and western blotting. The indirect co-culture model was established between SCC 25 cells and $\mathrm{CD} 14^{+}$monocytes (Fig. 7A). After co-culture for 3 and 6 days, RNA was extracted and real-time PCR was performed to detect gene expression changes. In monocytes cultured without CM of SCC25 cells, the transcriptional factor NFATc1 was expressed from day 3 until day 6 , and production of the proteinases MMP-9 and
CTSK increased on day 3 and day 6 (Fig. 7B), respectively. Similar expression trends for NFATc1 were found in monocytes after stimulation with CM of SCC25. For MMP-9 and CTSK, CM of SCC25 stimulated expression from the start of co-culture, which reached a maximum on day 6 . Changes in the protein levels of these targeted markers were further confirmed by western blotting (Fig. 7C and D).

CM of SCC25 cells induced more differentiation of human osteoclasts. To confirm the expression level changes of osteoclast markers in human monocytes, an osteoclast 

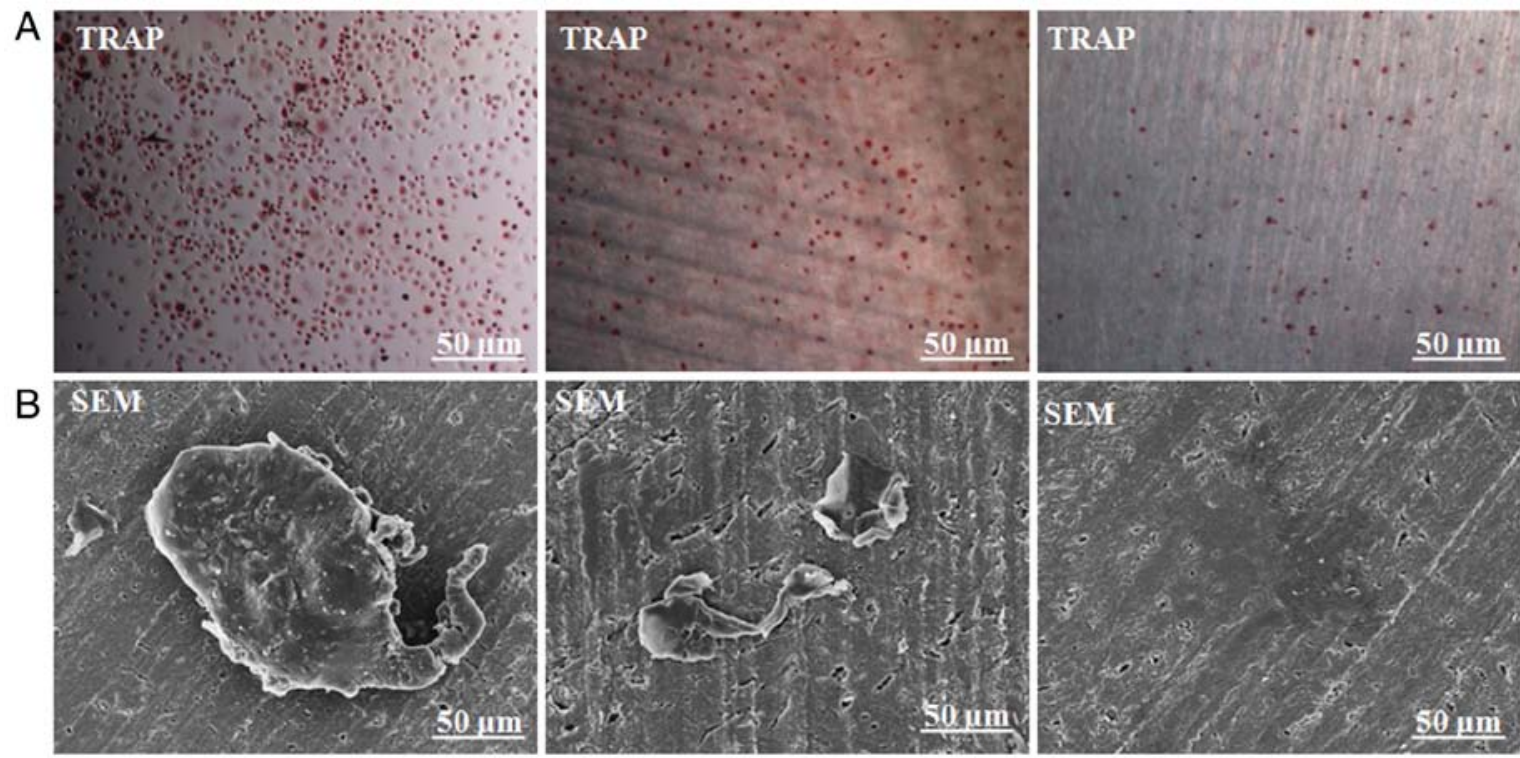

Figure 5. Bone resorption assay of mature osteoclasts. (A) TRAP staining of both cells with or without dentin slices revealed a normal color for TRAP; negative control dentin slice with only $\mathrm{CD}^{+} 4^{+}$monocytes. (B) A single giant osteoclast was found attached to the dentin slice. Negative control dentin slices with or without $\mathrm{CD} 14^{+}$monocytes were observed by SEM.
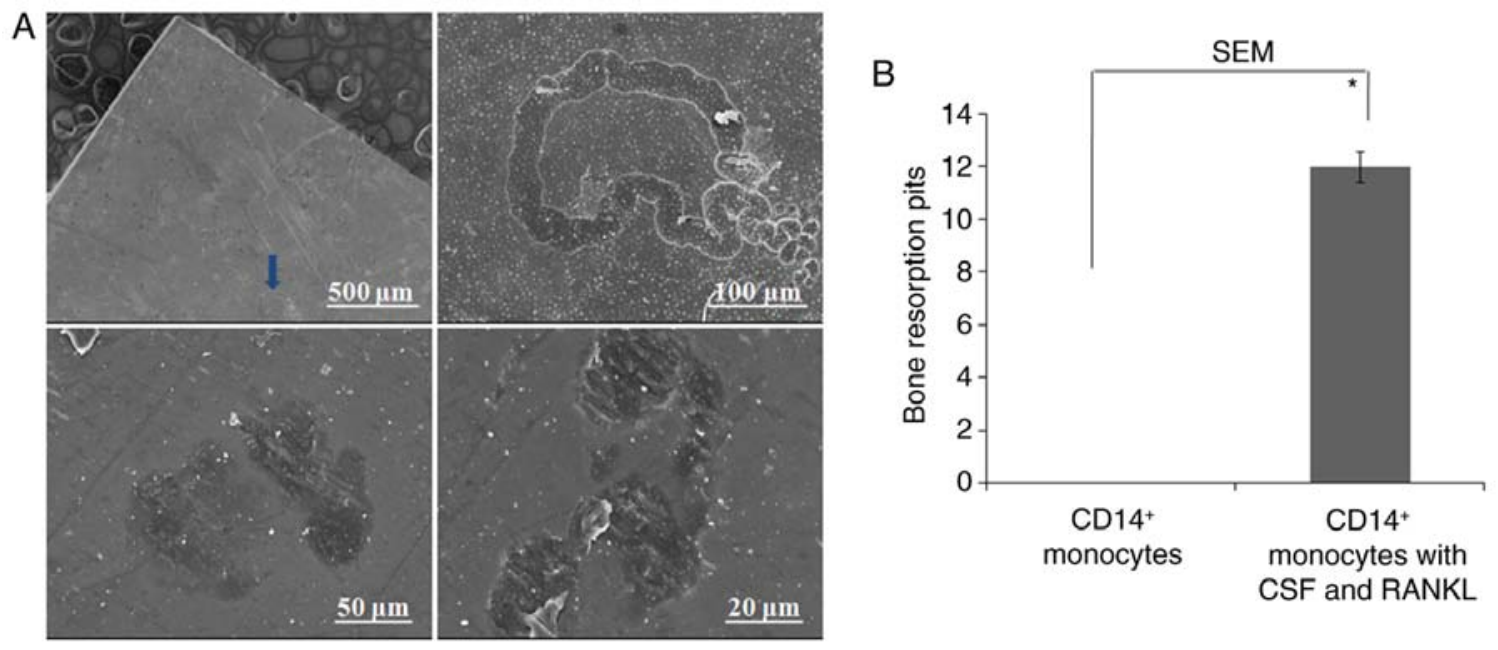

Figure 6. SEM confirmation of the bone resorption pits of dentin slices degraded by mature osteoclasts. (A) Different magnification was utilized to observe resorption pits, which were in the form of either single (arrow), small resorption tracks or discrete areas of lacunar excavations. (B) Quantification of bone resorption pits. Significant differences were found between these two groups of $\mathrm{CD} 14^{+}$monocytes and mature osteoclasts $\left({ }^{*} \mathrm{P}<0.05\right)$.

differentiation assay was further performed. Compared with the control group, more osteoclasts were observed on day 3 after stimulation with CM of SCC25 cells (Fig. 8). A great number of osteoclasts were observed on day 6 , and several differentiated osteoclasts were linked together. Similar results were obtained in IF, and larger numbers of F-actin rings were observed in both groups on days 3 and 6 (Fig. 8).

\section{Discussion}

The cultivation of osteoclasts in vitro is a pre-requisite for studying osteoclastogenesis (14). Current methods for isolating osteoclasts include using convenient immortal macrophage cell lines and primary mononuclear cells isolated from bone marrow (13). Raw 264.7 and THP1 cells are macrophage cell lines frequently used in osteoclast culture. Both are tumourderived cancer cell lines that can differentiate into mature osteoclasts upon induction with RANKL (15). However, cell lines often fail to mimic their primary counterparts (16), and not all observed changes are relevant to osteoclast development in vivo. Primary osteoclast culture from BMCs relies on the multiple differentiation ability of these cells. Cells from the bone marrow are heterogeneous and contain monocytes, blood cells, mesenchymal stem cells and other multipotent progenitor cells (17). Therefore, it is difficult to distinguish the exact cell types that differentiate into osteoclasts. In the present study, we isolated BMCs from the bone marrow of nude mice and attempted to differentiate them into osteoclasts. However, we did not obtain a sufficient number of osteoclasts for co-culture with tumour cells. The culture required a long 


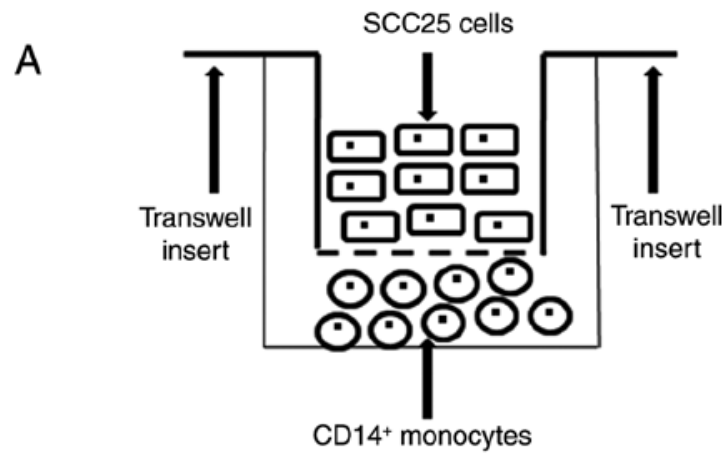

B

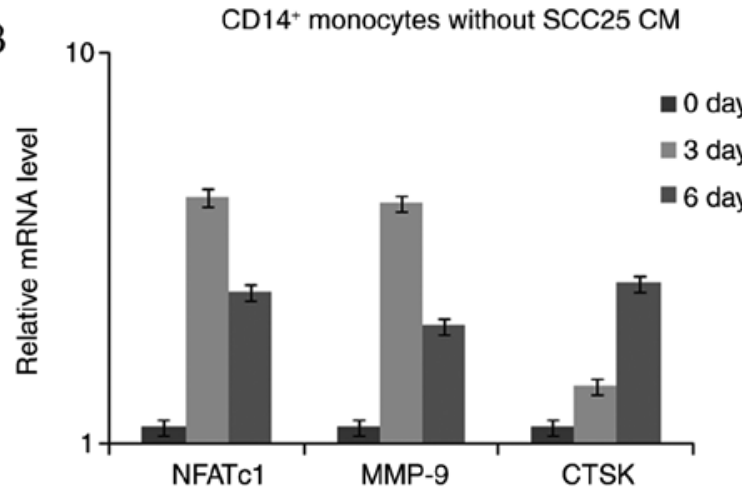

CD14+ monocytes without SCC25 CM

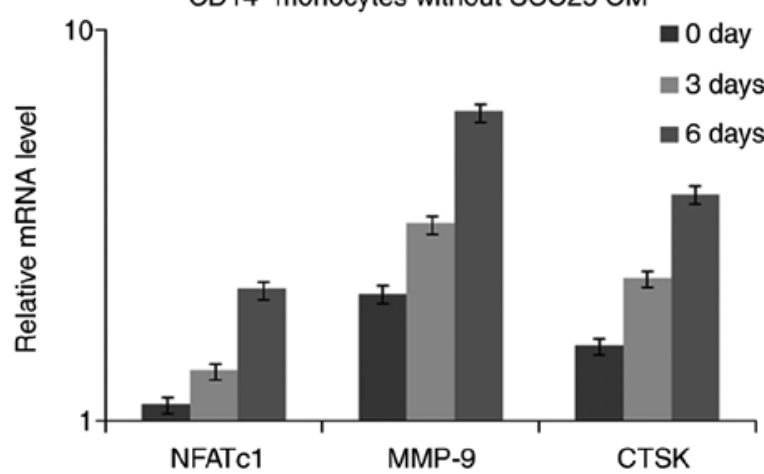

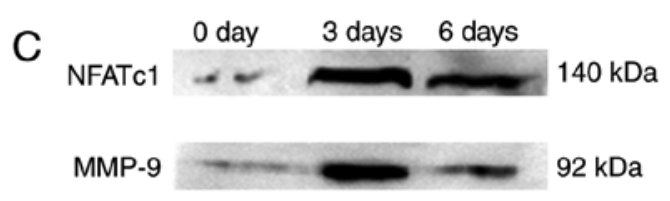

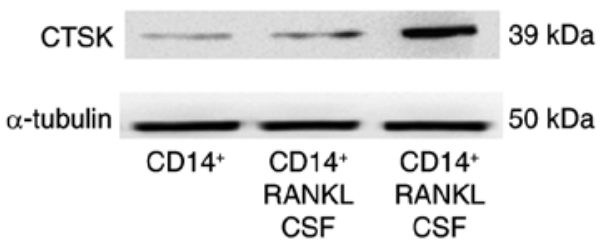

D 0 day 3 days 6 days
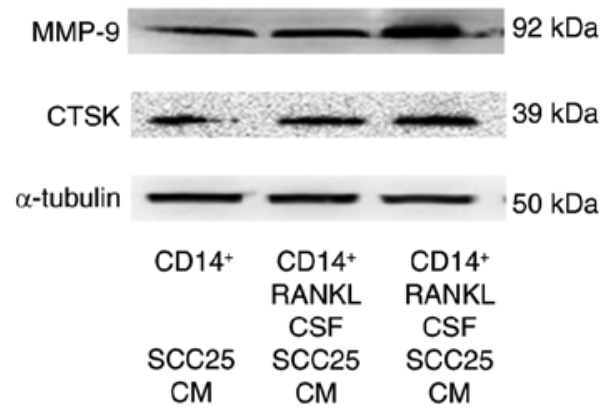

Figure 7. Expression changes in osteoclast markers detected by real-time PCR and western blotting. (A) Indirect cell co-culture model, SCC25 cells were added to the top of the Transwell insert, while CD14+ monocytes were plated to the bottom of 24-well plates. (B) For monocytes without stimulation from CM of SCC25 cells, NFATc1 was expressed on day 3 and until day 6; proteinases MMP-9 and CTSK were increased on days 3 and 6. Similar expression trends in NFATc1 were observed in monocytes after stimulation with SCC25 CM. For MMP-9 and CTSK, SCC25 CM stimulated their expression from the start of co-culture, which reached a maximum on day 6; (C and D) Protein level changes in NFATc1, MMP and CTSK were further confirmed by western blotting.

time and the cell number was limited, possibly because of deficient proliferative and differentiative ability of BMCs from nude mice. Thus, we used human PBMCs in the subsequent experiments.

Numerous studies have reported that osteoclasts can be generated from the PBMC populations, and these cells are commonly isolated by apheresis and density gradient centrifugation (18). However, this method is time-consuming and can result in a mixed red blood cell lysis solution (19). Currently, MACS is widely used to purify specific cell populations from PBMCs (20). In the present study, we used a specific centrifuge tube to obtain increased numbers of monocytes. As shown in Fig. 7, this centrifuge tube could enrich the monocytes as a single layer after centrifuging the blood, and was mainly composed of leukocytes, which could be easily removed into the collection tube. After washing and centrifugation, the collected monocytes were used for magnetic labeling with
MACS. This procedure was previously shown to be more specific and less time-consuming than other methods (19). Other factors affecting the osteoclast culture using MACS method include the freshness of peripheral blood, the centrifuging speed and temperature, the quality and quantity of collected leukocytes, and the handling skill of cell culture. Any of these factors can affect the abilities of cell proliferation and cell fusion, which may lead to the failure of osteoclast differentiation. In the present study differentiated osteoclasts were obtained from $\mathrm{CD} 14^{+}$monocytes after 6 days of culture. More importantly, a bone resorption assay was applied and dentin slices were observed after 20 days of continuous culture. Typical bone resorption pits in dentin slices were observed at different magnifications. Furthermore, a single giant osteoclast was observed and captured by SEM, which revealed the typical structure of mature osteoclasts. The ability to resorb the mineralized matrix is a crucial hallmark of these cells (21). 

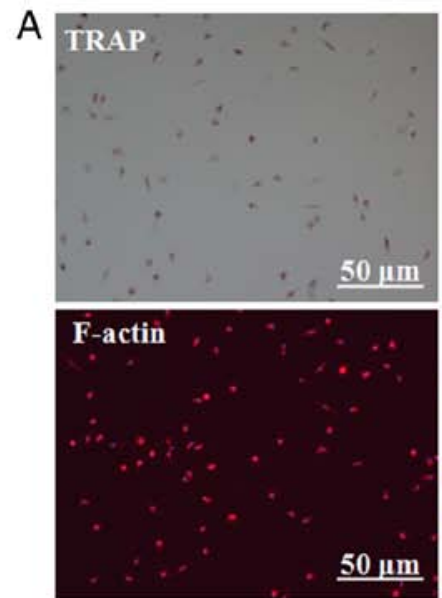

CD14+ monocytes with SCC25 CM
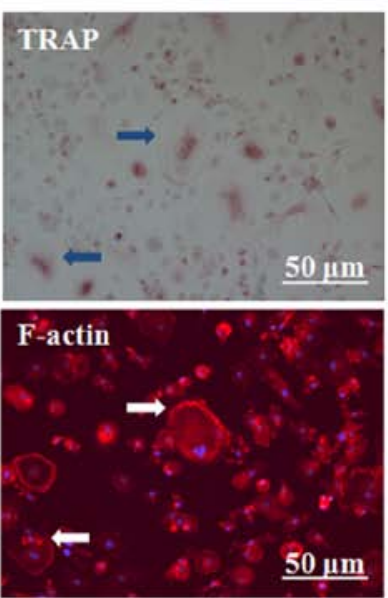

CD14+ monocytes with CSF and RANKL
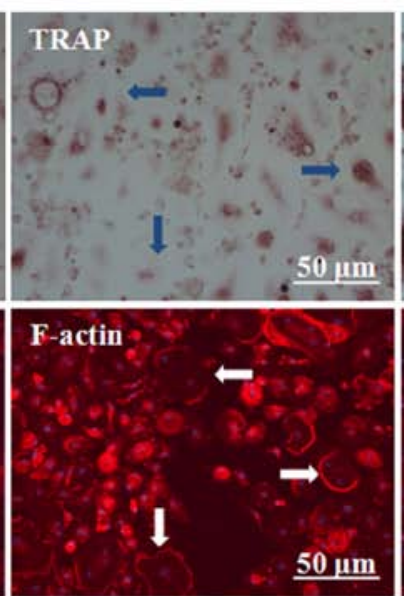

CD14+ monocytes with CSF and RANKL plus SCC25 CM (3 days)
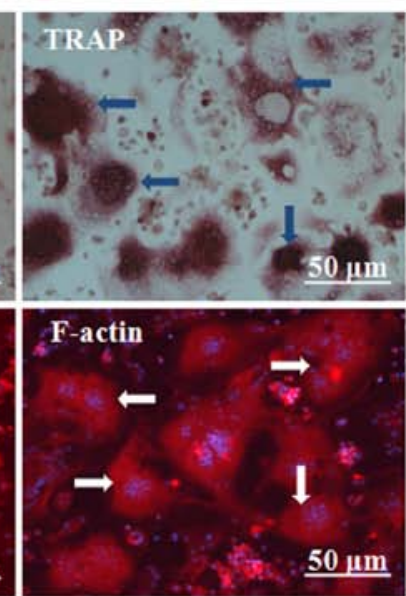

CD14+ monocytes

with CSF and RANKL

plus SCC25 CM ( 6 days)
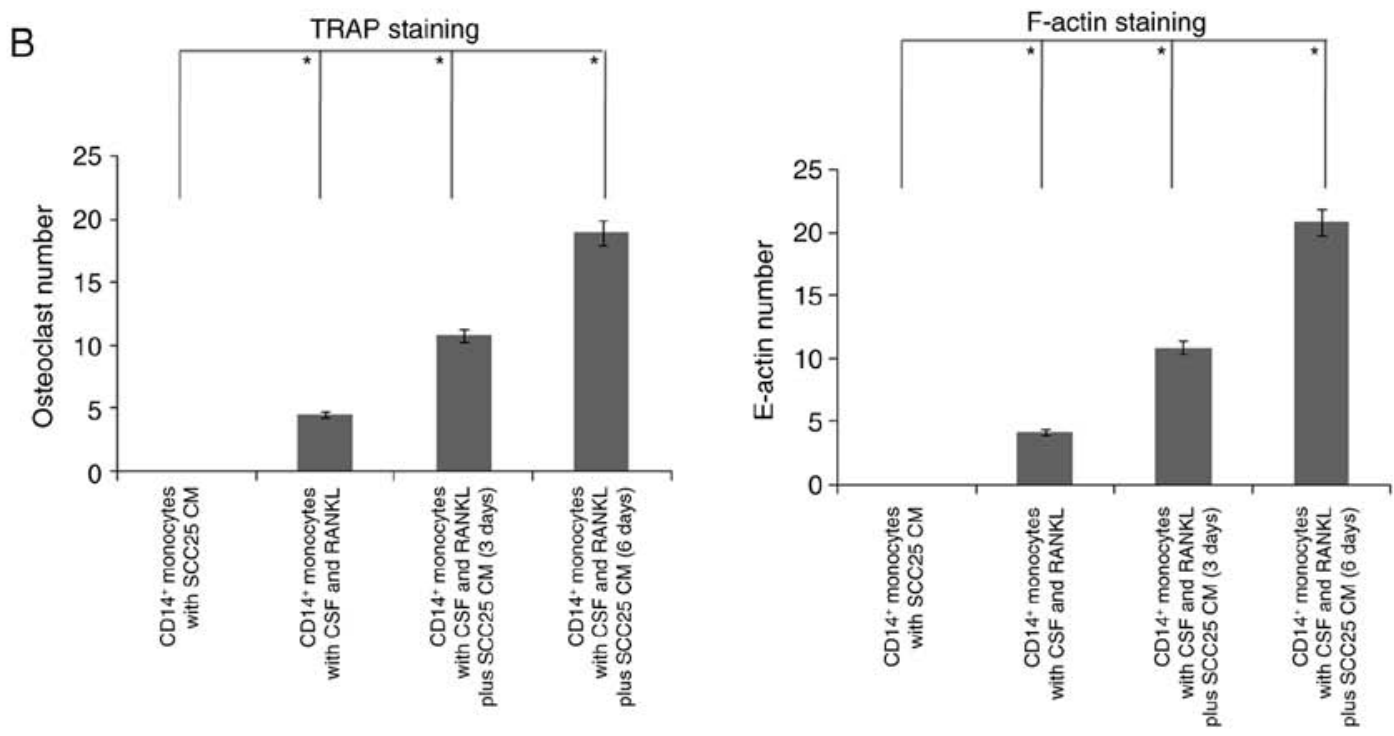

Figure 8. CM of SCC25 cells induces more differentiation of human osteoclasts. (A) Compared with the CD14 ${ }^{+}$monocytes cultured with cytokines CSF and RANKL, those cultured with CM of SCC25 cells yielded more osteoclasts on days 3 and 6 (arrow, TRAP). IF showed similar results, and greater numbers of F-actin rings were found in both groups on days 3 and 6 (arrow, F-actin). No osteoclasts were observed in the negative control group comprising CD14 monocytes only. Representative photos of TRAP and F-actin staining of osteoclasts. (B) Quantification of osteoclasts and F-actin. Significant differences were found between these 4 groups ( $(\mathrm{P}<0.05)$.

Bone resorption occurs in intricate and dynamic patterns, which facilitates the formation of complex bone shapes (1).

We next evaluated how tumour cells recruit osteoclast precursors to adjacent bone tissue. For this, we utilized Transwell inserts and established an indirect cell co-culture. The co-culture system is a useful tool for stimulating cell-cell communication, which occurs in the tumour microenvironment (22). The Transwell membrane allows secreted soluble factors to pass through, but prevents direct cell contact (22). A previous study showed that $10 \% \mathrm{CM}$ of SCC25 cells induces the formation of osteoclasts within 1 week of culture (9). However, expression of marker genes was not examined. Osteoclast markers, including TRAP, CTSK, c-FOS and NFATc1, are crucial for osteoclast differentiation $(13,23)$. The results of the present study showed that the master transcriptional regulator NFATc1 was present with the cytokines CSF and RANKL, and was increased on days 3 and 6. MMP-9 and CTSK were increased from days 3 to 6 . MMP-9 and CTSK, as the main proteinases, are typically observed during the late stage of osteoclast differentiation $(24,25)$. After stimulating with CM of SCC25 cells, NFATc1 still appeared on day 3 but was not observed in the initial culture. In contrast to expression patterns observed without SCC25 CM, MMP-9 and CTSK appeared and reached a maximum on day 6 . Additionally, $\mathrm{CM}$ of SCC25 stimulated MMP-9 and CTSK expression, indicating anabolic effects. The interactions between OSCC cells and osteoclast precursors promote the production of cytokines that stimulate osteoclastic cell function (22). The direct cell co-culture between monocytes and tumour cells will be the research plan of our future work. Based on published studies (26-28), the direct cell co-culture has been frequently utilized in studies seeking to understand the mechanisms of cancer progression and the preclinical trials of anticancer drugs (7). Since monocytes are derived from leukocytes of human peripheral blood, and the direct cell co-culture involves physical contact, whether these monocytes would identify and eliminate tumour 
cells as innate immune response is hard to know. Therefore, both the cell types and cell density are extremely important for establishing the direct co-culture model.

In summary, we observed different phenotypes of osteoclasts by establishing a bone invasion animal model and utilizing various primary cell cultures. Our results demonstrate that $\mathrm{CM}$ of OSCC cells can be used to induce the differentiation of osteoclasts from monocytes in the blood, with expression changes of osteoclast markers. This information can be used in studies of bone invasion by OSCC and may guide the development of biotherapies.

\section{Acknowledgements}

The present study was supported by the Medical Scientific Research Foundation of Guangdong Province (B2014164) and the National Natural Science Foundation of China (81500839).

\section{References}

1. Chambers TJ: The birth of the osteoclast. Ann NY Acad Sci 1192: 19-26, 2010.

2. Dou C, Cao Z, Yang B, Ding N, Hou T, Luo F, Kang F, Li J, Yang X, Jiang H, et al: Changing expression profiles of lncRNAs, mRNAs, circRNAs and miRNAs during osteoclastogenesis. Sci Rep 6: 21499, 2016.

3. Chen X, Wang Z, Duan N, Zhu G, Schwarz EM and Xie C: Osteoblast-osteoclast interactions. Connect Tissue Res 8: 1-9, 2017.

4. Strålberg F, Kassem A, Kasprzykowski F, Abrahamson M, Grubb A, Lindholm C and Lerner UH: Inhibition of lipopolysaccharide-induced osteoclast formation and bone resorption in vitro and in vivo by cysteine proteinase inhibitors. J Leukoc Biol 101: 1233-1243, 2017.

5. Martin TJ and Sims NA: RANKL/OPG; Critical role in bone physiology. Rev Endocr Metab Disord 16: 131-139, 2015.

6. Quan J, Johnson NW, Zhou G, Parsons PG, Boyle GM and Gao J: Potential molecular targets for inhibiting bone invasion by oral squamous cell carcinoma: A review of mechanisms. Cancer Metastasis Rev 31: 209-219, 2012

7. Quan J, Zhou C, Johnson NW, Francis G, Dahlstrom JE and Gao J: Molecular pathways involved in crosstalk between cancer cells, osteoblasts and osteoclasts in the invasion of bone by oral squamous cell carcinoma. Pathology 44: 221-227, 2012.

8. Quan J, Elhousiny M, Johnson NW and Gao J: Transforming growth factor- $\beta 1$ treatment of oral cancer induces epithelialmesenchymal transition and promotes bone invasion via enhanced activity of osteoclasts. Clin Exp Metastasis 30: 659-670, 2013.

9. Quan J, Morrison NA, Johnson NW and Gao J: MCP-1 as a potential target to inhibit the bone invasion by oral squamous cell carcinoma. J Cell Biochem 115: 1787-1798, 2014.

10. Khan UA, Hashimi SM, Bakr MM, Forwood MR and Morrison NA: Foreign body giant cells and osteoclasts are TRAP positive, have podosome-belts and both require OC-STAMP for cell fusion. J Cell Biochem 114: 1772-1778, 2013.

11. Livak KJ and Schmittgen TD: Analysis of relative gene expression data using real-time quantitative PCR and the $2(-\Delta \Delta \mathrm{C}(\mathrm{T}))$ method. Methods 25: 402-408, 2001.

12. Kim MS, Day CJ, Selinger CI, Magno CL, Stephens SR and Morrison NA: MCP-1-induced human osteoclast-like cells are tartrate-resistant acid phosphatase, NFATc1, and calcitonin receptor-positive but require receptor activator of NFkappaB ligand for bone resorption. J Biol Chem 281: 1274-1285, 2006.
13. Morrison NA, Day CJ and Nicholson GC: Dominant negative MCP-1 blocks human osteoclast differentiation. J Cell Biochem 115: 303-312, 2014.

14. Xiong Q, Zhang L, Zhan S, Ge W and Tang P: Investigation of proteome changes in osteoclastogenesis in low serum culture system using quantitative proteomics. Proteome Sci 14: 8, 2016.

15. Pivetta E, Wassermann B, Bulian P, Steffan A, Colombatti A, Polesel J and Spessotto P: Functional osteoclastogenesis: The baseline variability in blood donor precursors is not associated with age and gender. Oncotarget 6: 31889-31900, 2015.

16. Penolazzi L, Lolli A, Sardelli L, Angelozzi M, Lambertini E, Trombelli L, Ciarpella F, Vecchiatini R and Piva R: Establishment of a 3D-dynamic osteoblasts-osteoclasts co-culture model to simulate the jawbone microenvironment in vitro. Life Sci 152: 82-93, 2016.

17. Szpalski C, Barbaro M, Sagebin F and Warren SM: Bone tissue engineering: current strategies and techniques - part II: Cell types. Tissue Eng Part B Rev 18: 258-269, 2012.

18. Zhang $M$ and Huang B: The multi-differentiation potential of peripheral blood mononuclear cells. Stem Cell Res Ther 3: 48, 2012.

19. Mayer A, Lee S, Lendlein A, Jung F and Hiebl B: Efficacy of $\mathrm{CD} 14^{+}$blood monocytes/macrophages isolation: Positive versus negative MACS protocol. Clin Hemorheol Microcirc 48: 57-63, 2011.

20. Sprangers S, Schoenmaker T, Cao Y, Everts V and de Vries TJ: Integrin $\alpha \mathrm{M} \beta 2$ is differently expressed by subsets of human osteoclast precursors and mediates adhesion of classical monocytes to bone. Exp Cell Res 350: 161-168, 2017.

21. Castillo LM, Guerrero CA and Acosta O: Expression of typical osteoclast markers by PBMCs after PEG-induced fusion as a model for studying osteoclast differentiation. J Mol Histol 48: $169-185,2017$.

22. Teixeira LN, de Castro Raucci LM, Alonso GC, Coletta RD, Rosa AL and de Oliveira PT: Osteopontin expression in co-cultures of human squamous cell carcinoma-derived cells and osteoblastic cells and its effects on the neoplastic cell phenotype and osteoclastic activation. Tumour Biol 37: 12371-12385, 2016.

23. Hemingway F, Cheng X, Knowles HJ, Estrada FM, Gordon S and Athanasou NA: In vitro generation of mature human osteoclasts. Calcif Tissue Int 89: 389-395, 2011.

24. Khan UA, Hashimi SM, Khan S, Quan J, Bakr MM, Forwood MR and Morrison NM: Differential expression of chemokines, chemokine receptors and proteinases by foreign body giant cells (FBGCs) and osteoclasts. J Cell Biochem 115: 1290-1298, 2014.

25. Khan UA, Hashimi SM, Bakr MM, Forwood MR and Morrison NA: CCL2 and CCR2 are essential for the formation of osteoclasts and foreign body giant cells. J Cell Biochem 117: 382-389, 2016

26. Shabo I, Midtbö K, Andersson H, Åkerlund E, Olsson H, Wegman P, Gunnarsson C and Lindström A: Macrophage traits in cancer cells are induced by macrophage-cancer cell fusion and cannot be explained by cellular interaction. BMC Cancer 15 : 922,2015

27. Mercatali L, Spadazzi C, Miserocchi G, Liverani C, De Vita A, Bongiovanni A, Recine F, Amadori D and Ibrahim T: The effect of everolimus in an in vitro model of triple negative breast cancer and osteoclasts. Int J Mol Sci 1: 17, 1827, 2016.

28. Low HB, Png CW, Li C, Wang Y, Wong SB and Zhang Y: Monocyte-derived factors including PLA2G7 induced by macrophage-nasopharyngeal carcinoma cell interaction promote tumor cell invasiveness. Oncotarget 7: 55473-55490, 2016.

This work is licensed under a Creative Commons Attribution-NonCommercial-NoDerivatives 4.0 International (CC BY-NC-ND 4.0) License. 